Trauma Berufskrankh 2007 - 9 [Suppl 2]:S245-S248 DOI 10.1007/s10039-006-1202-3

Online publiziert: 14. März 2007

(c) Springer Medizin Verlag 2007
A.P. Verheyden · N. Koch · S. Katscher · A. Hölzl

Klinik für Unfall-, Orthopädische und Wirbelsäulenchirurgie,

Ortenau-Klinikum Lahr-Ettenheim, Lahr

\title{
Versorgungsstrategien bei Verletzungen der Lendenwirbelsäule
}

\section{Hintergrund und Fragestellung}

Eine operative Intervention nach Wirbelkörperfrakturen wird in der Regel in Abhängigkeit von neurologischen Defiziten, Art der Instabilität, Ausmaß der Deformität und des Allgemeinzustands des $\mathrm{Pa}$ tienten vorgenommen $[6,10]$. Aufgrund des in den letzten Jahren erheblich breiter gewordenen Spektrums operativer Interventionsmöglichkeiten werden die Ergebnisse der unterschiedlichen Therapieoptionen bei Lendenwirbelkörperfrakturen verglichen, um die Indikationsstellung zu erleichtern.

Zunächst wird auf die biomechanischen Besonderheiten der lumbalen Wirbelsäule und die daraus resultierenden Konsequenzen für die Behandlung von Verletzungen der LWS eingegangen. Es werden aktuelle Aspekte der Operationstechnik bei dorsalem, ventralem und kombiniertem [2] operativem Vorgehen dargestellt, wobei besonderer Schwerpunkt auf die minimalinvasiven Zugänge gelegt wird. Abschließend werden Aspekte für die Zukunft andiskutiert.

\section{Lendenwirbelsäule}

\section{Biomechanik}

Im Stehen befindet sie sich aufgrund ihrer physiologischen Lordorsierung komplett ventral der Tragachse. Daraus folgt, dass v. a. die dorsalen Strukturen erheblich druckbelastet sind. Auf den ventralen Anteilen der Wirbelkörper dagegen ist diese Belastung geringer. Deshalb be- steht bei primär kyphotisch deformierten Wirbelkörpern im LWS-Bereich auch bei konservativer Behandlung ein gewisses Korrekturpotenzial.

\section{Korrekturverlust}

Vergleicht man Angaben in der Literatur über posttraumatische Fehlstellungen und Korrekturverlust, muss unterschieden werden, ob der betroffene Wirbelkörper oder der Wirbelkörper einschließlich der hauptbetroffenen Bandscheibe - in der Regel der oberen - oder der Wirbelkörper mit beiden benachbarten betroffenen Bandscheiben gemessen worden ist [3]. In der Gesamtliteratur findet man zwar viele Angaben für einen Korrekturverlust an der thorakolumbalen Wirbelsäule [4], eine Differenzierung zwischen thorakaler bzw. lumbaler Wirbelsäule und thorakolumbalem Übergang erfolgt dabei in der Regel jedoch nicht $[1,7,10]$.

\section{Studiendesign und Untersuchungsmethoden}

Ziel dieser Arbeit ist die differenzierte Darstellung der Behandlungsindikationen von Lendenwirbelsäulenverletzungen. Grundlage ist neben der aktuellen Literatur eine 2-Jahres-Studie von 1994/95, in der bei Wirbelköperfrakturen ab AO-Klassifikation A3.1 eine ausschließlich dorsale Fixateur-interne-Instrumentation ohne Spongiosaplastik oder Facettengelenkarthrodese mit konservativ funktioneller Behandlung verglichen wurden. Der durchschnittliche Nachuntersu- chungszeitpunkt betrug mehr als 12 Monate nach Metallentfernung des Fixateur interne.

\section{Ergebnisse}

Bezüglich des Wirbelkörpers zeigte sich an der LWS bei konservativer Therapie ein Ausheilen in etwas geringerer Kyphosierung als zum Zeitpunkt direkt nach dem Unfall. Nach operativer Therapie war der Wirbelkörper wieder in fast anatomischer Stellung. Bezog man allerdings die nächstobere Bandscheibe mit in die Messung ein, zeigte sich insgesamt ein Korrekturverlust von unter $5^{\circ}$ nach operativer Therapie. Bei Einbeziehung beider Zwischenwirbelräume in die Berechnung war der Korrekturverlust noch geringer.

Zusammenfassend konnte man feststellen, dass es an der Lendenwirbelsäule vergleichsweise geringe Probleme mit dem Korrekturverlust gibt. Nach konservativer Therapie traten insgesamt weniger Korrekturverluste auf als nach operativer Behandlung. Einen möglichen Grund hierfür sehen wir in einer nicht zusätzlich stattfindenden Schädigung der Bandscheiben durch die Immobilisation im Fixateur interne [5].

Somit empfehlen wir bei stabilen Wirbelkörperfrakturen Typ A1.2 nach Magerl im LWS-Bereich mit einer Wirbelkörperkyphosierung von unter $20^{\circ}$ eine konservativ funktionelle Therapie.

Die wichtigste Devise bei der operativen Therapie insbesondere im Bereich der Lendenwirbelsäule ist, so wenig Bewegungssegmente wie möglich aufzugeben. 


\section{Wirbelsäule}

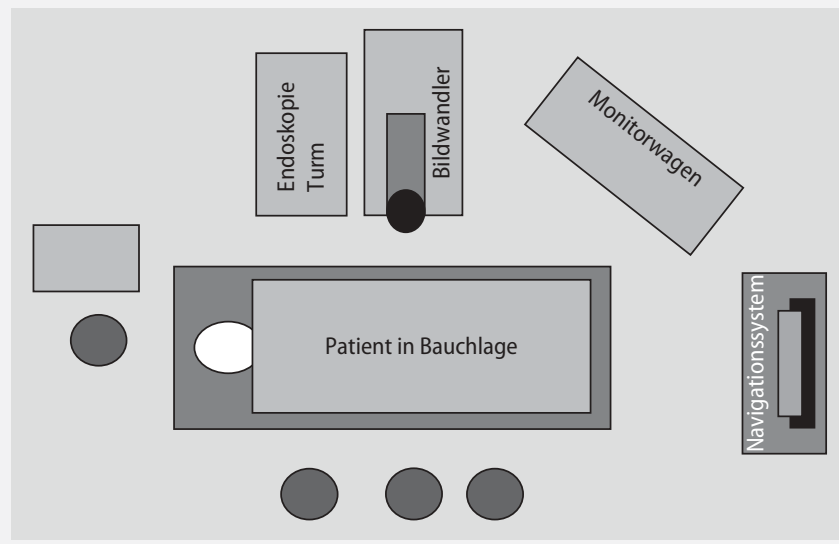

Abb. $1 \Delta$ Aufbau im OP

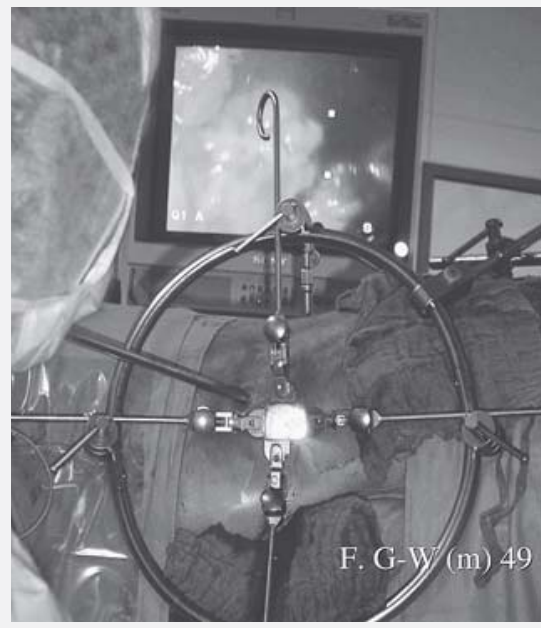

Abb. $3 \Delta$ Ventral retroperitonealer Zugang in Bauchlage mit Synframe ${ }^{\circledast}$

Abb. $4 \triangleright$ Instabile L3-Fraktur beim alten Menschen

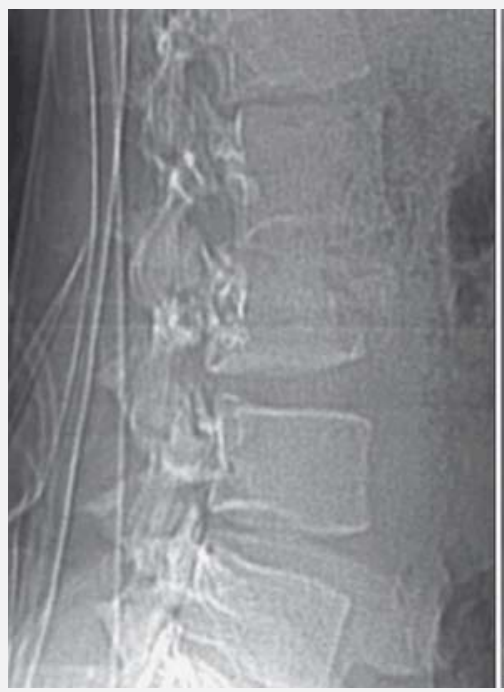

Frakturen vom Typ A3.1 nach Magerl sollten nach Möglichkeit nur monosegmental fusioniert werden.

\section{Operative Techniken in der Diskussion}

\section{Ventrale Technik}

Wir bevorzugen die minimalinvasive, ventrale Technik [13, 14].

\section{Implantat und Instrumentation}

Bei ausschließlich ventraler Instrumentation sind die Anforderungen an ein ventrales Implantat erheblich:

- Es sollte winkelstabil und monosegmental zu implantieren sein.
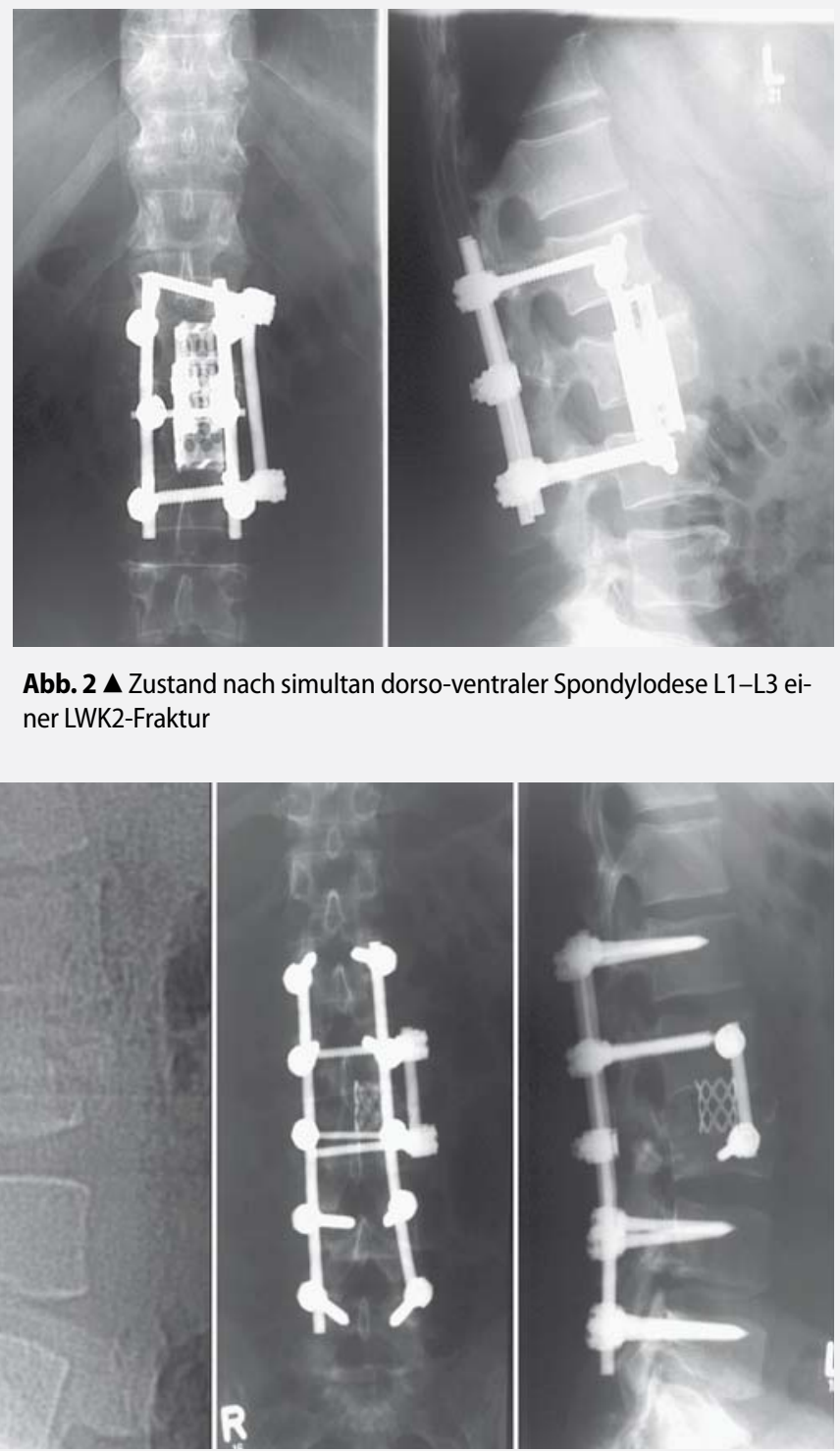

Abb. $2 \Delta$ Zustand nach simultan dorso-ventraler Spondylodese L1-L3 einer LWK2-Fraktur
- Es sollte eine hohe Lastaufnahme und eine Reposition mit Hilfe des Implantats ermöglichen.

- Es sollte die Instrumentation in minimalinvasiver Technik realisieren lassen.

Sehr hilfreich haben sich dabei die expandierenden Cages erwiesen. Sie haben die intraoperativen Repositionsmöglichkeiten erheblich verbessert. Zur Neutralisation der Zugkräfte empfehlen wir allerdings gerade an der Lendenwirbelsäule immer die Kombination mit Einstabinstrumentation, auch wenn bereits eine dorsale Instrumentation vorliegt. Bei ausschließlich ventralem Vorgehen sollte der Cage immer mit einer biomechanisch ausreichend stabilen ventralen Instrumenta- tion kombiniert werden. Für „stand alone cages" sehen wir in der Traumatologie an der lumbalen Wirbelsäule keine Indikation.

\section{Biomechanische Grundvoraussetzung}

Für eine ausschließlich ventrale Instrumentation muss der Zuggurtungsmechanismus der dorsalen knöchernen und ligamentären Wirbelsäulenstrukturen intakt sein. Es muss also mit Sicherheit eine A-Verletzung vorliegen. Dies kann einerseits durch präoperative MRT-Untersuchungen mit ausreichender Sicherheit festgestellt werden, andererseits kann man mit geeigneten Instrumentarien auch intraoperativ die Stabilität der dorsalen Bandstrukturen überprüfen. 
Da wir die Patienten primär auch bei geplantem ausschließlich ventralem Vorgehen in Bauchlage operieren (• Abb. 1), haben wir die Möglichkeit, bei entsprechender Instabilität ohne erhöhten Aufwand wie Umlagern eine dorsale Instrumentation zu ergänzen ( $\bullet$ Abb. 2).

\section{Lagerung, Zugang und Operation}

Da das Spontanalignment der Wirbelsäule in Bauchlage am besten ist, simultan dorsale und ventrale Zugänge durchgeführt und auch bei ausschließlich ventralem Vorgehen die Navigation eingesetzt werden können, führen wir die Eingriffe an der Lendenwirbelsäule in der Regel in Bauchlage durch. Wir verwenden dabei eine minimalinvasive endoskopisch gestützte Technik mit einem Hautschnitt von etwa $4 \mathrm{~cm}$ Länge lateral in Projektion auf den betroffenen Wirbelkörper und dann sorgfältiger retroperitonealer Präparation mit Hilfe eines Retraktorsystems (Synframe ${ }^{\varpi}$, Fa. Synthes) (• Abb. 3).

Die Navigation erweist sich nicht nur bei der Identifikation der knöchernen Strukturen als sehr hilfreich. Insbesondere die Lage der Gefäße im unteren Lendenwirbelbereich lässt sich mit ihrer Hilfe einfach identifizieren und so die Sicherheit des operativen Vorgehens erhöhen [12].

Zum Set-up im Operationssaal sei auf - Abb. 1 verwiesen.

\section{Dorsale Instrumentation}

Hierbei favorisieren wir zurzeit nach einiger Erfahrung mit perkutanen Techniken einen Hautschnitt in der medialen Linie und erst auf Faszienebene eine gezielte Inzision der Faszie in Verlängerung der Pedikelachse ohne Ablösung der Muskulatur. Diese Technik erfordert zwar einen längeren Hautschnitt, ist letztendlich u. E. aber weniger traumatisierend für die Muskulatur wie die rein perkutanen Methoden, da die Längsträger unter taktiler Kontrolle weichteilschonend in die Schicht zwischen Lamina und kurzer Rückenmuskulatur eingesetzt werden können.
Trauma Berufskrankh 2007 - 9 [Suppl 2]:S245-S248 DOI 10.1007/s10039-006-1202-3

(c) Springer Medizin Verlag 2007

A.P. Verheyden · N. Koch · S. Katscher · A. Hölzl Versorgungsstrategien bei Verletzungen der Lendenwirbelsäule

\section{Zusammenfassung}

Der vorliegende Beitrag befasst sich mit der differenzierten Indikationsstellungen bei Verletzungen der Lendenwirbelsäule und der technischen Durchführung der minimalinvasiven Operationsverfahren. Auf der Basis biomechanischer Grundlagen der Lendenwirbelsäule und den Ergebnissen einer Studie, die konservative und operative Therapien verglich, wird ein Behandlungskonzept vorgestellt. Die technische Durchführung auch simultaner dorso-ventraler Eingriffe an der Lendenwirbelsäule in Bauchlage wird erläutert. An der LWS gibt es vergleichsweise geringe Probleme mit dem Korrekturverlust.

Nach konservativer Therapie treten insgesamt weniger Korrekturverluste auf als nach operativer Behandlung. Wir empfehlen bei Wirbelkörperfrakturen Typ A1.2 der LWS mit einer Kyphosierung $<20^{\circ}$ eine konservativ funktionelle Therapie. Instabile A-Frakturen und A-Frakturen mit einer Kyphose $>20^{\circ}$ werden von uns bevorzugt in minimalinvasiver ventraler Technik versorgt.

\section{Schlüsselwörter}

Wirbelfrakturen · Lendenwirbelsäule · Korrekturverlust · Bauchlage · Simultan dorso-ventrale Stabilisierung

\section{Treatment strategies in injuries to the lumbar spine}

\section{Abstract}

This paper deals with the specific indications for different treatments in cases of spinal fractures in the lumbar segment and with the techniques applied in current operative procedures. We present a concept of treatment that is based on the biomechanics of the spine and the results of a comparative study of conservative and operative treatments. The technical implementation of simultaneous dorsal-ventral procedures on the lumbar spine with the patient in lying prone are explained. Relatively few problems involving loss of correction are encountered in the lumbar spine. On balance, loss of correction oc- curs less frequently after a conservative therapy than after invasive interventions. We recvertebral fractures with kyphotic deformation by $<20^{\circ}$. In the case of unstable fractures and type $A$ fractures associated with kyphotic deformations by $>20^{\circ}$ we prefer to apply a minimally invasive technique and use a ventral approach.

\section{Keywords}

Spinal injury · Lumbar spine - Loss of correction in the spine $\cdot$ Prone position · Simultaneous dorsal-ventral stabilisation ommend noninvasive-therapy for type A1.2 


\section{Alter Patient}

Bei den relativ selten auftretenden instabilen Wirbelkörperfrakturen der Lendenwirbelsäule im Alter ist die Devise

- langstreckig dorsal instrumentieren,

- kurzstreckig ventral fusionieren.

Auf diese Weise lässt sich auch bei osteoporotischen Knochen und instabilen Situationen eine frühfunktionelle Mobilisierung erreichen ( $\bullet$ Abb. 4).

Bei den stabilen Frakturformen der Lendenwirbelsäule und älteren Patienten haben Vertebro- und Kyphoplastie das Spektrum der minimalinvasiven Therapien erheblich erweitert. Insbesondere Letztere ermöglicht eine gute Reposition der Wirbelkörper, ohne die Bandscheiben zusätzlich zu schädigen, und einen Erhalt des Bewegungsausmaßes. Die Indikation zur Kyphoplastie sehen wir allerdings erst ab einem biologischem Alter von 6o Jahren. Die Verwendung bioresorbierbarer Zemente für jüngere Patienten zeigte bisher keine ausreichenden Ergebnisse.

\section{Ausblick}

Aussichten für die Zukunft könnten im Bereich der Lendenwirbelsäule bei geeignetem Frakturmuster gezielte Osteosynthesen des verletzten Wirbelkörpers sein, ggf. sogar in Kombination mit einer Bandscheibenprothese.

Ein anderer Aspekt ist die Frage der Metallentfernung nach dorsaler Instrumentation. Zur Minimierung der sekundären Bandscheibenschädigung durch den Fixateur interne entfernen wir diesen nach abgeschlossener Konsolidierung in der Regel bereits nach 3 Monaten.

Ein weiterer Aspekt für die Zukunft wäre die Anpassung der Elastizität der Implantate individuell an den Typ des Patienten und die Art der Verletzung

\section{Fazit für die Praxis}

An der Lendenwirbelsäule ist bei A-Frakturen mit initialer Kyphose von weniger als $20^{\circ}$ eine konservative Therapie indiziert. Ausnahmen sind nur die osteoporotischen Frakturen des alten Menschen, bei welchen in Abhängigkeit von der Be- schwerdesymptomatik Vertebro- oder Kyphoplastie sinnvoll sind.

Alle instabilen A-Frakturen und A-Frakturen mit initialer Kyphose von mehr als $20^{\circ}$ werden bei uns bevorzugt minimalinvasiv möglichst kurzstreckig von ventral versorgt. B- und C-Verletzungen werden in Abhängigkeit von der Bandscheibenverletzung entweder dorsal oder kombiniert dorso-ventral versorgt.

\section{Korrespondierender Autor}

\section{Prof. Dr. A.P. Verheyden}

Klinik für Unfall-, Orthopädische und Wirbelsäulenchirurgie, Ortenau-Klinikum Lahr-Ettenheim, Klosterstraße 19, 77933 Lahr akhil.verheyden@le.ortenau-klinikum.de

Interessenkonflikt. Es besteht kein Interessenkonflikt. Der korrespondierende Autor versichert, dass keine Verbindungen mit einer Firma, deren Produkt in dem Artikel genannt ist, oder einer Firma, die ein Konkurrenzprodukt vertreibt, bestehen. Die Präsentation des Themas ist unabhängig und die Darstellung der Inhalte produktneutral.

\section{Literatur}

1. Böhler L (1938) Wirbelbrüche und Wirbelverrenkungen. In: Böhler L (Hrsg) Technik der Knochenbrucbehandlung, Bd 1. Maurich, Wien, S 249-3691

2. Dimar JR, Wilde PH, Glassman SD et al. (1996) Thoracolumbar burst fractures treated with combined anterior and posterior surgery. Am J Orthop 25: 159-165

3. Eysel P, Rompe JD, Hopf C et al. (1994) Die Bedeutung der Bandscheibe für den Repositionsverlust operativ stabilisierter Frakturen der Rumpfwirbelsäule. Unfallchirurg 97: 451-457

4. Kaneda K, Taneichi H, Abumi K et al. (1997) Anterior decompression and stabilisation with the Kaneda Device for thoracolumbar burst fractures associated with neurological deficits. J Bone Joint Surg Am 79: 69-83

5. Katscher S, Verheyden AP, Gonschorek O et al. (2003) Thorakolumbale Wirbelfrakturen nach konservativer und operativer Behandlung. Abhängigkeit des Korrekturverlustes von der Höhenlokalisation. Unfallchirurg 106: 20-27

6. Knop C, Blauth M, Bühren V et al. (2000) Operative Behandlung von Verletzungen des thorakolumbalen Übergangs. Teil 2: Operationen und röntgenologische Befunde. Unfallchirurg 103: 1032-1047

7. Magnus G (1934) Die Wirbelsäule. In: König F, Magnus $\mathrm{G}$ (Hrsg) Handbuch der gesamten Unfallheilkunde, Bd 4. Enke, Stuttgart, S 1-46

8. Muehlbauer M, Pfisterer W, Eyb R et al. (2000) Minimally invasive anterior approach for lumbar corporectomy and anterior reconstruction. J Neurosurg 93: 161-167

9. Onimus M, Papin P, Gangloff S (1996) Extraperitoneal approach to the lumbar spine with video assistance. Spine 21: 2491-2494
10. Resch H, Rabl M, Klampfer H et al. (2000) Operative vs. konservative Behandlung von Frakturen des thorakolumbalen Übergangs. Unfallchirurg 103: 281-288

11. Trojan E (1972) Langfristige Ergebnisse von 200 Wirbelbrüchen der Brust- und Lendenwirbelsäule ohne Lähmungen. Unfallmed Berufskrankh 66: 122-134

12. Verheyden AP, Hölzl A (2003) Erfahrungen zu Kosten und Nutzen der Navigation in der Wirbelsäulenchirurgie. Trauma Berufskrankh [Suppl 2] 6 : 197-201

13. Verheyden AP, Katscher S, Gonschorek $O$ et al. (2002) Die endoskopisch assistierte minimal invasive Rekonstruktion der vorderen thorakolumbalen Wirbelsäule in Bauchlage. Unfallchirurg 105: 873-880

14. Verheyden AP, Hoelzl A, Lill H et al. (2004) The endoscopically assisted simultaneous postero-anterior reconstruction of the thoracolumbar spine in prone position. Spine J 4: 540-549 